\title{
ASYMPTOTIC BEHAVIOR OF MEROMORPHIC FUNCTIONS WITH EXTREMAL SPREAD II
}

\section{ALBERT EDREI and WOLFGANG H. J. FUCHS}

Introduction. This paper is a continuation of [3]. In view of our constant reference to specific formulae, lemmas and theorems of [3], we write [A] instead of [3] and use, with their obvious meaning, notations such as

$$
\text { (12.1 [A]), Lemma } 4.1 \text { [A], Theorem } 3 \text { [A]. }
$$

The notational conventions of $[\mathrm{A}]$ are adopted without modifications and strictly adhered to.

We supplement Theorems 1, 2 and 3 of [A] by the more detailed information contained in the theorems of the present paper.

In everything that follows

(i) $f(z)$ is a meromorphic function of lower order $\mu$

$$
0<\mu<+\infty \text {, }
$$

satisfying the hypotheses ES at a sequence $\left\{r_{m}\right\}_{m=1}^{\infty}$ of Pólya peaks of order $\mu$ of $T(r)=$ $=T(r, f)$;

(ii) $2 \beta$ is the spread of $\infty$ at the peaks $\left\{r_{m}\right\}$ and

$$
\delta(\infty, f)=1-\cos \beta \mu, \quad 0<\beta \leqq \pi / 2 \mu ;
$$

(iii) $I_{m}(s)=\left\{r: r_{m} e^{-s}<r \leqq r_{m} e^{s}\right\}(s>0), \Lambda(s)=\bigcup_{m=1}^{\infty} I_{m}(s)$.

We first prove

Theorem 4. Let $f(z)$ satisfy the hypotheses ES and let the positive quantities $s, \varepsilon, \eta(\eta<(1 / 2) \min (\beta, \pi-\beta))$ be given.

It is then possible to determine a real sequence $\left\{\omega_{m}\right\}$ and a point set $\mathscr{E}=\mathscr{E}_{m}(s, \varepsilon)$ which may be enclosed in disks with sum of diameters not exceeding $\varepsilon e^{-s} r_{m}$ and having the following properties.

The research was supported by grants from the National Science Foundation; GP-33175 for the first author and GP-28251 for the second author. 
I. If

(1)

$$
r \in I_{m}(s), \quad r e^{i \theta} \notin \mathscr{E}_{m}(s, \varepsilon), \quad m>m_{0},
$$

then

$$
\log \left|f\left(r e^{i\left(\theta+\omega_{m}\right)}\right)\right| \leqq \varepsilon T(r) \quad(\beta-\eta \leqq|\theta| \leqq \pi)
$$

and

$$
|\log | f\left(r e^{i\left(\theta+\omega_{m}\right)}\right)|-\pi \mu \sin \mu(\beta-|\theta|) T(r)| \leqq \varepsilon T(r) \quad(\eta \leqq|\theta| \leqq \beta-\eta) .
$$

II. If $v_{m}$ denotes the number of zeros of $f(z)$ in the sector

$$
\left\{r e^{i \theta}: r \in I_{m}(s),\left|\theta-\omega_{m}\right| \leqq \beta-\eta\right\},
$$

and $\pi_{m}$ the number of poles in the sector

we have

$$
\left\{r e^{i \theta}: r \in I_{m}(s), \eta \leqq\left|\theta-\omega_{m}\right| \leqq \pi\right\},
$$

$$
v_{m}+\pi_{m}=o\left(T\left(r_{m}\right)\right) \quad(m \rightarrow \infty) .
$$

Our next two theorems show that, under the hypotheses ES, two very different types of behavior may occur in the sectors

$$
\left\{r \in I_{m}(s), \beta<\theta-\omega_{m}<2 \pi-\beta\right\} .
$$

Theorem 5. Suppose that the hypotheses of Theorem 4 are satisfied and that

Then

$$
\pi-\beta>\pi / 2 \mu \text {. }
$$

$$
\begin{gathered}
\log \left|f\left(r e^{i\left(\theta+\omega_{m}\right)}\right)\right| \geqq-\varepsilon T(r) \\
\left(r \in I_{m}(s), \beta+\eta \leqq|\theta| \leqq \pi ; r e^{i\left(\omega_{m}+\theta\right)} \notin \tilde{\mathscr{E}}_{m}(s, \varepsilon, \eta) ; m>m_{0}\right) .
\end{gathered}
$$

Here $\tilde{\mathscr{E}}_{m}(s, \varepsilon, \eta)$ is an exceptional set which may be covered by a finite number of disks the sum of whose diameters is less than $\varepsilon e^{-s} r_{m}$.

If $\tilde{v}_{m}$ denotes the number of zeros of $f(z)$ in

we have

$$
\left\{r e^{i \theta}: r \in I_{m}(s), \beta+\eta \leqq\left|\theta-\omega_{m}\right| \leqq \pi\right\},
$$

$$
\tilde{v}_{m}=o\left(T\left(r_{m}\right)\right) \quad(m \rightarrow \infty) .
$$

Remark I. Since $\mu \beta \leqq \pi / 2$, the condition (5) is satisfied for all $\mu>1$.

If $1 / 2<\mu \leqq 1$, (5) is equivalent to

$$
\cos \mu \beta>\sin \mu \pi \text {. }
$$

Remark II. If (5) is satisfied, the relations (4) and (7) hold simultaneously. Hence, almost all the zeros of $f(z)$, in the annulus $z \in I_{m}(s)$, lie in one or the other of the two angles

$$
\left|\theta-\omega_{m}+\beta\right|<\eta, \quad\left|\theta-\omega_{m}-\beta\right|<\eta .
$$

It is natural to expect that there will be approximately the same number of zeros in each of these angles. This is in fact true. 
More precisely, given $m, \xi(0<\xi<1)$ and $\eta(0<\eta<\beta)$, denote by $n^{+}(u)$ the number of zeros of $f(z)$ in the sector

$$
\xi r_{m}<|z| \leqq u, \quad \omega_{m}+\beta-\eta \leqq \arg z \leqq \omega_{m}+\beta+\eta .
$$

If in (9) the restrictions on the argument are replaced by

$$
\omega_{m}-\beta-\eta \leqq \arg z \leqq \omega_{m}-\beta+\eta,
$$

we obtain a symmetrical sector and denote by $n^{-}(u)$ the analogous counting function of the zeros of $f(z)$ in this sector.

With the above definitions we may state our

Asymptotic symmetry. Let the assumptions of Theorem 5 be satisfied. Then

I. For every $s>0$,

$$
\frac{n(r, 1 / f)}{T(r)} \rightarrow \mu \quad(r \rightarrow \infty, r \in \Lambda(s)) .
$$

II. Given $t$ such that

we have

$$
0<t<\min (1-\xi, \sin \eta)
$$

$$
\frac{n^{+}\left(r_{m}(1+t)\right)-n^{+}\left(r_{m}(1-t)\right)}{T\left(r_{m}\right)} \rightarrow \frac{\mu}{2}\left\{(1+t)^{\mu}-(1-t)^{\mu}\right\} \quad(m \rightarrow \infty) .
$$

The relation (12) still holds if $n^{+}$is replaced by $n^{-}$.

It is convenient to prove the asymptotic symmetry in the preceding form (a brief sketch of the proof will be found in Section 13). Generalizations are immediate and we may deduce from the above result the asymptotic equality of the number of zeros in the two sectors for which $|z| \in I_{m}(s)$ and $\arg z$ is restricted as in (9) and (10).

Theorem 6. If the hypotheses of Theorem 4 are satisfied and (5) does not hold, then (6) and (7) need not be true.

Assume that (6) does not hold; it is then possible to find an infinite set $\mathscr{M}$ of positive integers such that for $m \in \mathscr{M}, r \in I_{m}(s), \beta+\eta \leqq|\theta| \leqq \pi$

$$
\log \mid f\left(r e^{\left.i \theta+i \omega_{m}\right)} \mid<-K T\left(r_{m}\right) \quad(0<K=K(s, \eta)=\text { const. }),\right.
$$

outside an exceptional set $\mathscr{E}_{m}(s, \varepsilon, \eta)$ which can be enclosed in disks with sum of diameters $<\varepsilon e^{-s} r_{m}$.

Theorems 4,5 and 6 contain fairly detailed information about $f(z)$. It is easy to deduce from them

Theorem 7. Let the meromorphic function $f(z)$ satisfy the hypotheses ES then $f(z)$ has at most one finite deficient value. There are no finite deficient values if $f(z)$ satisfies the additional condition (5). 
Remark II shows that, in the annuli $|z| \in I_{m}(s)$ and under the hypothesis (5), almost all the zeros lie in the angles (8). On the other hand, by Theorem 4 , in the same annuli, almost all poles lie in $\left|\theta-\omega_{m}\right|<\eta$. This is the 'fork pattern' of zeros and poles exhibited by the function $\mathscr{H}(z)$ of the introduction of [A].

Hence Theorem 5 describes completely the situation in the case characterized by (5) and is, in an obvious sense, "best possible".

If (5) is not satisfied and if $1 / 2<\mu \leqq 1$, we have, by Remark I,

and the line

$$
\cos \mu \beta \leqq \sin \mu \pi
$$

intersects the arc

$$
y=\cos \mu \beta
$$

$$
x^{2}+y^{2}-2 x y \cos \mu \pi=\sin ^{2} \mu \pi, \quad x \geqq 0, \quad y \geqq 0
$$

at

$$
x_{0}=\cos \mu(\pi-\beta) \quad y_{0}=\cos \mu \beta .
$$

Then, as was shown in [1, pp. 244-249],

$$
\begin{cases}f(z)=g\left(x_{0}^{1 / \mu} z\right) / g\left(-y_{0}^{1 / \mu} z\right) & \left.\left(g(z)=\prod_{1}^{\infty}\left(1+z / n^{1 / \mu}\right), 1 / 2<\mu<1\right)\right), \\ f(z)=\prod_{2}^{\infty}\left(1+\frac{z}{n \log ^{2} n}\right) \quad(\mu=1),\end{cases}
$$

have deficient zeros and poles with

The spreads

$$
1-\delta(\infty, f)=\cos \mu \beta, \quad 1-\delta(0, f)=\cos \mu(\pi-\beta) .
$$

$$
\sigma(\infty, f)=2 \beta, \quad \sigma(0, f)=2(\pi-\beta),
$$

are both extremal. But the zeros of $f(z)$ are all real and negative, so that we cannot have the fork pattern. It is also easy to verify that, for the above functions, (13) holds for all large $r$.

It is now clear that Theorem 6 accurately describes the asymptotic behavior in all cases not covered by Theorem 5 . In order to pass from Theorem 4 to Theorem 5 it is necessary to make appropriate use of the condition (5).

To perform this step we use a modification of Carleman's identity. Our version introduces a "central logarithmic mean" which is expressed in terms of the expected contributions of zeros, poles and boundary values of $|f(z)|$. The presence of the central mean (analogous to the central value in Jensen's formula) is useful for our purpose. We prove

Carleman's identity for a sector. Let $g(z)$ be meromorphic in the sector

$$
D: t e^{-\sigma} \leqq|z| \leqq t e^{\sigma} \quad(0<\sigma), \quad|\arg z| \leqq \pi / 2 c \quad(c>1 / 2) .
$$


Let

$$
\Xi(\theta)=\frac{c}{2 \pi}\left\{\int_{t e^{-\sigma}}^{t} \log \left|g\left(e^{i \theta} y\right)\right|\left(\frac{y^{c} e^{\sigma c}}{t^{c}}-\frac{t^{c}}{y^{c} e^{\sigma c}}\right) d y / y\right.
$$

$$
\left.+\int_{t}^{t e^{\sigma}} \log \left|g\left(e^{i \theta} y\right)\right|\left(\frac{t^{c} e^{\sigma c}}{y^{c}}-\frac{y^{c}}{t^{c} e^{\sigma c}}\right) d y / y\right\}
$$

and

$$
\eta\left(|a| e^{i \varphi}\right)= \begin{cases}\left(\frac{|a|^{c} e^{\sigma c}}{t^{c}}-\frac{t^{c}}{|a|^{c} e^{\sigma c}}\right) \cos (c \varphi) & \left(t e^{-\sigma} \leqq|a|<t\right), \\ \left(\frac{t^{c} e^{\sigma c}}{|a|^{c}}-\frac{|a|^{c}}{t^{c} e^{\sigma c}}\right) \cos (c \varphi) & \left(t \leqq|a| \leqq t e^{\sigma}\right) .\end{cases}
$$

Denote by a a zero of $g(z)$ in $D$ and by $b$ one of its poles.

Then

$$
\begin{aligned}
& \frac{c}{\pi}\left(e^{\sigma c}+e^{-\sigma c}\right) \int_{-\pi / 2 c}^{\pi / 2 c} \log \left|g\left(t e^{i \theta}\right)\right| \cos (c \theta) d \theta \\
= & \frac{c}{\pi} \int_{-\pi / 2 c}^{\pi / 2 c} \log \left|g\left(t e^{-\sigma} e^{i \theta}\right) g\left(t e^{\sigma} e^{i \theta}\right)\right| \cos (c \theta) d \theta+\Xi(-\pi / 2 c)+\Xi(\pi / 2 c) \\
+ & \Sigma_{b} \eta(b)-\Sigma_{a} \eta(a),
\end{aligned}
$$

where $\Sigma_{b}$ denotes summation over all poles $b$ of $g(z)$ in $D$ and $\Sigma_{a}$ summation over all zeros a of $g(z)$ in $D$.

Our last result concerns entire functions of lower order $\mu>1 / 2$. If we confine our attention to such functions, the class for which Paley's relation is realized in extremal form coincides exactly with the class satisfying the hypotheses ES. More precisely:

Theorem 8. Let $f(z)$ be an entire function which satisfies the hypotheses ES at some sequence $\left\{r_{m}\right\}_{m}$ of peaks of order $\mu$ of $T(r, f)$.

Then, necessarily,

$$
\lim _{m \rightarrow \infty} \frac{\log M\left(r_{m}, f\right)}{T\left(r_{m}, f\right)}=\pi \mu .
$$

Conversely, if (17) holds at a sequence $\left\{r_{m}\right\}_{m}$ of peaks of order $\mu>1 / 2$, then $f(z)$ must satisfy the hypotheses ES at the same sequence of peaks.

The fact that (17) follows from the hypotheses ES is an almost immediate consequence of Theorem 3 [A].

To obtain the converse, use the above relation (17) in Lemma 11.2 [A] to prove (with the notations of $[\mathrm{A}, \S 16]$ )

as well as $(15[\mathrm{~A}])$.

$$
L_{m}^{-}=o\left(T\left(r_{m}\right)\right), \quad \tilde{L}_{m}=o\left(T\left(r_{m}\right)\right),
$$


After these points have been established, all the necessary arguments are simple special cases of those in Sections 2, 3 and 4 below.

1. Recall of $[\mathrm{A}]$ and definitions. Let $\eta(0<\eta<\min (1, \beta, \pi-\beta))$ and $s=(1 / \eta)$ be given. Then, by Theorem 1 [A], we may assert that, for

$$
r_{m} e^{-s}<t \leqq r_{m} e^{s}, \quad m>m_{0}(\eta),
$$

we have

$$
\begin{aligned}
\frac{T(t)}{t^{\mu}}=\frac{T\left(r_{m}\right)}{r_{m}^{\mu}}(1+\eta(t)) \quad\left(|\eta(t)| \leqq \eta, m>m_{0}(\eta, s)\right), \\
|N(t, f)-T(t) \cos \mu \beta|<\eta T(t), \\
|n(t, f)-T(t) \mu \cos \mu \beta|<\eta T(t) .
\end{aligned}
$$

Let $p_{m}$ be the number of poles of $f(z)$ in the sector

$$
\left\{r e^{i \theta}: r_{m} e^{-s}<t \leqq r_{m} e^{s}, \eta \leqq\left|\theta-\omega_{m}\right| \leqq \pi\right\}
$$

and $z_{m}$ the number of zeros $f(z)$ in the sector

$$
\left\{r e^{i \theta}: r_{m} e^{-s}<t \leqq r_{m} e^{s},\left|\theta-\omega_{m}\right| \leqq \beta-\eta\right\} .
$$

By Theorem $3[\mathrm{~A}]$ we know that the conditions (1.1) and a suitable choice of $m_{0}(\eta)$ imply

$$
p_{m}+z_{m} \leqq \eta^{2} T\left(r_{m}\right) \quad\left(m>m_{0}(\eta)\right) .
$$

The simple "diagonalization" described in [A] enables us to select a positive sequence $\left\{\tilde{\eta}_{m}\right\}$ and an associated sequence $\left\{s_{m}\right\}$ defined by

and such that

$$
s_{m}=1 / \tilde{\eta}_{m},
$$

(ii) if

$$
\begin{gathered}
\tilde{\eta}_{m} \rightarrow 0, \quad s_{m} \rightarrow \infty \quad(m \rightarrow \infty) ; \\
r_{m}^{\prime} \leqq e^{-s_{m}} r_{m} \leqq t \leqq e^{s_{m}} r_{m} \leqq r_{m}^{\prime \prime},
\end{gathered}
$$

then (1.2), (1.3), (14) hold with $\eta$ replaced by $\tilde{\eta}_{m}$; as to (1.7) it takes the form

Redefine $r_{m}^{\prime}, r_{m}^{\prime \prime}$ as

$$
\left(p_{m}+z_{m}\right) s_{m} \leqq \tilde{\eta}_{m} T\left(r_{m}\right) .
$$

$$
r_{m}^{\prime}=e^{-s_{m}} r_{m}, \quad r_{m}^{\prime \prime}=e^{s_{m}} r_{m},
$$

and discard the former quantities $r_{m}^{\prime}, r_{m}^{\prime \prime}$. With the new notations, $p_{m}$ and $z_{m}$ in (1.9) denote, respectively, the number of poles of $f(z)$ in the sector

$$
\mathscr{P}_{m}=\left\{r e^{i \theta}: r_{m}^{\prime}<r \leqq r_{m}^{\prime \prime}, \omega_{m}+\tilde{\eta}_{m} \leqq \theta \leqq 2 \pi+\omega_{m}-\tilde{\eta}_{m}\right\},
$$

and the number of zeros of $f(z)$ in the sector

$$
\mathscr{Z}_{m}=\left\{r e^{i \theta}: r_{m}^{\prime}<r \leqq r_{m}^{\prime \prime}, \omega_{m}-\beta+\tilde{\eta}_{m} \leqq \theta \leqq \omega_{m}+\beta-\tilde{\eta}_{m}\right\} .
$$


Let $\left\{b_{j}\right\}_{j},\left\{a_{j}\right\}_{j}$ denote, respectively, the poles and the zeros of $f(z)$ and define the polynomials

$$
P_{m}(z)=\prod_{b_{j} \in \mathscr{P}_{m}}\left(1-z / b_{j}\right), \quad Z_{m}(z)=\prod_{a_{j} \in \mathscr{P}_{m}}\left(1-z / a_{j}\right),
$$

of respective degrees $p_{m}, z_{m}$.

It follows immediately from our definitions that

$$
\varphi_{m}(z)=\log |f(z)|+\log \left|P_{m}(z)\right| \quad\left(z \in \mathscr{P}_{m}\right)
$$

is subharmonic, and that

$$
\psi_{m}(z)=\varphi_{m}(z)-\log \left|Z_{m}(z)\right|
$$

is harmonic in

$$
\mathscr{D}_{m}=\left\{r e^{i \theta}: r_{m}^{\prime}<r \leqq r_{m}^{\prime \prime}, \omega_{m}+\tilde{\eta}_{m} \leqq \theta \leqq \omega_{m}+\beta-\tilde{\eta}_{m}\right\}
$$

as well as in the symmetrical sector $\mathscr{D}_{m}^{*}$ with $\theta$ restricted by

$$
2 \pi+\omega_{m}-\beta+\tilde{\eta}_{m} \leqq \theta \leqq 2 \pi+\omega_{m}-\tilde{\eta}_{m} .
$$

Lemma 1.1. I. Let $s>1$ be given and let

$$
Q_{m}(z)=\Pi(1-z / a)
$$

be a polynomial of degree $q_{m}$ such that each one of its zeros a satisfies the conditions

$$
r_{m} e^{-s-1} \leqq|a| \leqq r_{m} e^{s+1} .
$$

Then, if

$$
|z|=r \leqq r_{m} e^{s+1},
$$

we have

$$
\frac{1}{2 \pi} \int_{-\pi}^{\pi}|\log | Q_{m}\left(r e^{i \theta}\right)|| d \theta \leqq 10 s q_{m} .
$$

II. For the polynomials $P_{m}(z)$ and $Z_{m}(z)$ defined by (1.12), the restriction

implies

$$
|z| \leqq r_{m} e^{s_{m}}
$$

$$
\begin{gathered}
\log \left|P_{m}(z)\right|<\eta_{m} T\left(r_{m}\right), \quad \log \left|Z_{m}(z)\right|<\eta_{m} T\left(r_{m}\right), \\
\frac{1}{2 \pi} \int_{-\pi}^{\pi}\left\{|\log | P_{m}\left(r e^{i \theta}\right)||+|\log | Z_{m}\left(r e^{i \theta}\right)||\right\} d \theta<\eta_{m} T\left(r_{m}\right) .
\end{gathered}
$$

III. Given $\varepsilon \quad(0<\varepsilon<1)$ it is possible to find sets $\mathscr{E}_{m}(s, \varepsilon)$ such that

(i) $\mathscr{E}_{m}(s, \varepsilon)$ is the union of finitely many disks with sum of diameters $\leqq(1 / 2) \varepsilon e^{-s} r_{m}$;

(ii) the condition

implies

$$
z_{m} \notin \mathscr{E}_{m}(s, \varepsilon)
$$

$$
\log \left|P_{m}(z)\right|>-\eta_{m} T\left(r_{m}\right), \quad \log \left|Z_{m}(z)\right|>-\eta_{m} T\left(r_{m}\right),
$$

and

$$
\log \left|Q_{m}(z)\right| \geqq-q_{m}(7 s+\log (1 / \varepsilon)) .
$$


Proof of I. In view of (1.16) and (1.17)

$$
\left|\frac{z}{a}\right| \leqq e^{2 s+2}
$$

and hence

$$
\log \left|Q_{m}(z)\right| \leqq q_{m} \log \left(1+e^{2 s+2}\right) \leqq 5 s q_{m} .
$$

By Jensen's formula and (1.23)

$$
\frac{1}{2 \pi} \int_{-\pi}^{\pi}+\log \left|\frac{1}{Q_{m}\left(r e^{i \theta}\right)}\right| d \theta \leqq \frac{1}{2 \pi} \int_{-\pi}^{\pi}+\log \left|Q_{m}\left(r e^{i \theta}\right)\right| d \theta \leqq 5 s q_{m},
$$

and (1.18) follows.

Proof of II. The above proof is easily adapted to yield (1.19) and (1.20). For instance, to treat $P_{m}(z)$, replace, in (1.23), $s$ by $s_{m}$ and $q_{m}$ by $p_{m} T\left(r_{m}\right)$. Since, by (1.9), $s_{m} p_{m} \rightarrow 0$, the analogue of $(1.23)$ is

$$
\log \left|P_{m}(z)\right| \leqq 5 s_{m} p_{m} T\left(r_{m}\right)=\eta_{m} T\left(r_{m}\right) \quad\left(|z| \leqq r_{m} e^{s_{m}}\right) .
$$

The remaining inequalities of assertion II are now obvious. [For sake of clarity, we remind the reader of the convention that $\left\{\eta_{m}\right\}$ is a sequence of real numbers tending to 0 as $m \rightarrow \infty$, not always the same at each occurrence; $\eta_{m}$ is independent of the variables $t, r, \theta$.]

Proof of III. It will be sufficient to examine $Q_{m}(z)$. By the Boutroux-Cartan lemma and (1.16)

$$
\left|Q_{m}(z)\right|=\prod_{a} \frac{|z-a|}{|a|} \geqq\left(\frac{h}{4 e}\right)^{q_{m}} r_{m}^{-q_{m}} e^{-q_{m}(s+1)}
$$

outside circles the sum of whose diameters does not exceed $h$. Choose

$$
h=\frac{1}{2} \varepsilon e^{-s} r_{m}
$$

It follows that, outside a set $\mathscr{E}_{m}(s, \varepsilon)$, we find

and (1.22) follows.

$$
\log \left|Q_{m}(z)\right| \geqq q_{m}(\log \varepsilon-2 s-5),
$$

2. Upper bounds for $\varphi_{m}(z)$, lower bounds for $\psi_{m}(z)$. A subharmonic function $\Omega(z)$ cannot exceed the average of $\Omega$ over the area of a disk with center at $z$.

We use this elementary property in the following form.

Lemma 2.1. I. Let $\Omega(\zeta)$ be subharmonic in the sector
$r e^{-s-1} \leqq|\zeta| \leqq r e^{s+1}$
$(s \geqq 0)$
$\theta_{1} \leqq \theta=\arg \zeta \leqq \theta_{2}$
$\left(0<\theta_{2}-\theta_{1}<2 \pi\right)$. 
Assume that, for some $\delta>0$,

$$
\frac{1}{2 \pi} \int_{r e^{-s-1}}^{r e^{s+1}} x d x \int_{\theta_{1}}^{\theta_{2}} \Omega^{+}\left(x e^{i \varphi}\right) d \varphi \leqq \delta .
$$

Then, given $\eta\left(0<\eta<\min \left\{\left(\theta_{\mathrm{z}}-\theta_{1}\right) / 2, \pi / 6\right\}\right)$, we have

$$
\Omega^{+}(\zeta) \leqq \frac{2 \delta \exp (2 s)}{r^{2} \sin ^{2} \eta}
$$

throughout the sector

$$
r e^{-s} \leqq|\zeta| \leqq r e^{s}, \quad \theta_{1}+\eta \leqq \theta \leqq \theta_{2}-\eta .
$$

II. Assume that $\Omega(\zeta)$ is harmonic in the sector (2.1) and that, in addition to (2.2), we have

$$
\frac{1}{2 \pi} \int_{r e^{-s-1}}^{r e^{s+1}} x d x \int_{\theta_{1}}^{\theta_{2}}\left\{-\Omega\left(x e^{i \varphi}\right)\right\}^{+} d \varphi \leqq \delta
$$

Then

$$
|\Omega(\zeta)| \leqq \frac{2 \delta e^{2 s}}{r^{2} \sin ^{2} \eta}
$$

throughout the sector (2.4).

Proof. Let $t e^{i \theta}$ lie in the sector defined by (2.4). It is then clear that the whole disk

$$
\Gamma=\left\{\zeta:\left|\zeta-t e^{i \theta}\right| \leqq t \sin \eta\right\}
$$

lies in the sector defined by (2.1). Hence, in view of (2.2)

$$
\frac{1}{2 \pi} \iint_{\Gamma} \Omega^{+}(\zeta) d A \leqq \frac{1}{2 \pi} \int_{t(1-\chi)}^{t(1+\chi)} x d x \int_{\theta_{1}}^{\theta_{2}} \Omega^{+}\left(x e^{i \varphi}\right) d \varphi \leqq \delta \quad(\chi=\sin \eta),
$$

where $d A$ is the element of area. By the behavior of the average of the subharmonic function $\Omega^{+}(\zeta)$, we now have

$$
\Omega^{+}(\zeta) \leqq \frac{1}{\pi t^{2} \sin ^{2} \eta} \iint_{\Gamma} \Omega^{+}(\zeta) d A \leqq \frac{2 \delta}{t^{2} \sin ^{2} \eta},
$$

and (2.3) follows.

To prove assertion II notice that, in addition to (2.7),

$$
\{-\Omega(\zeta)\}^{+} \leqq \frac{2 \delta}{t^{2} \sin ^{2} \eta}
$$

From (2.7) and (2.8) we deduce (2.6) and thus complete the proof of the lemma.

Lemma 2.2. Let $s>0$ and $\eta$

$$
0<\eta<\min (\beta / 2, \pi-\beta, \pi / 6),
$$

be given and let $z$ be confined to the annuli

$$
e^{-s-1} r_{m} \leqq|z| \leqq e^{s+1} r_{m} .
$$


Then, the following inequalities hold uniformly in $z$ :

$$
\begin{gathered}
\varphi_{m}(z) \leqq \frac{2 e^{\mu+2}}{\sin ^{2}(\eta / 2)}\left(r / r_{m}\right)^{\mu} T\left(r_{m}\right) \quad\left(\eta \leqq \theta-\omega_{m} \leqq 2 \pi-\eta, m>m_{0}(\eta, s)\right), \\
\varphi_{m}(z) \leqq \eta_{m} T\left(r_{m}\right) \quad\left(\beta+\eta \leqq \theta-\omega_{m} \leqq 2 \pi-\beta-\eta, \eta_{m}>0\right), \\
\psi_{m}(z) \geqq-\eta_{m} T\left(r_{m}\right) \quad\left(\eta \leqq\left|\theta-\omega_{m}\right| \leqq \beta-\eta, \eta_{m}>0\right) .
\end{gathered}
$$

Proof. Let $\theta_{1}<\theta_{2}<\theta_{1}+2 \pi$ and let

$$
J=\left\{\theta: \theta_{1} \leqq \theta \leqq \theta_{2}\right\} .
$$

Then, our definitions (1.13), (1.14) and the inequality (1.20) imply

$$
\begin{gathered}
\frac{1}{2 \pi} \int_{\theta_{1}}^{\theta_{2}} \varphi_{m}^{+}\left(r e^{i \theta}\right) d \theta \leqq m(r, f ; J)+\eta_{m} T\left(r_{m}\right) \quad\left(r \in I_{m}(s+2)\right), \\
\frac{1}{2 \pi} \int_{\theta_{1}}^{\theta_{2}}\left\{-\psi_{m}\left(r e^{i \theta}\right)\right\}^{+} d \theta \leqq m(r, 1 / f ; J)+\eta_{m} T\left(r_{m}\right) \quad\left(r \in I_{m}(s+2)\right) .
\end{gathered}
$$

In particular, from (2.14) we deduce

$$
\frac{1}{2 \pi} \int_{\theta_{1}}^{\theta_{2}} \varphi_{m}^{+}\left(x e^{i \theta}\right) d \theta \leqq T(x)+\eta_{m} T\left(r_{m}\right) \leqq 2\left(x / r_{m}\right)^{\mu} T\left(r_{m}\right) \quad\left(x \in I_{m}(s+2), m>m_{0}\right),
$$

$$
\frac{1}{2 \pi} \int_{r e^{-1}}^{r e} x d x \int_{\theta_{1}}^{\theta_{2}} \varphi_{m}^{+}\left(x e^{i \theta}\right) d \theta \leqq e^{\mu+2} r^{2}\left(r / r_{m}\right)^{\mu} T\left(r_{m}\right) \quad\left(r \in I_{m}(s+1), m>m_{0}\right) .
$$

Since $\varphi_{m}(z)$ is subharmonic in $\mathscr{P}_{m}$ (defined by (1.10)) we may use Lemma 2.1 with

$$
\theta_{1}=\omega_{m}+\eta / 2, \quad \theta_{2}=2 \pi+\omega_{m}-\eta / 2
$$

and thus deduce (2.11) from (2.16).

To prove (2.12) and (2.13) we return to [A] and focus our attention on Lemma 16.1 [A] from which we immediately deduce

$$
\begin{gathered}
\int_{r_{m} e^{-s-2}}^{r_{m} e^{s+2}} m\left(r, f ;\left[\omega_{m}+\beta, \omega_{m}+2 \pi-\beta\right]\right) X\left(r, r_{m}\right) d r / r \leqq \eta_{m} T\left(r_{m}\right), \\
\int_{r_{m} e^{-s-2}}^{r_{m} e^{s+2}} m\left(r, 1 / f ;\left[\omega_{m}-\beta, \omega_{m}+\beta\right]\right) X\left(r, r_{m}\right) d r / r \leqq \eta_{m} T\left(r_{m}\right)
\end{gathered}
$$

with

$$
\frac{1}{r^{2}} X\left(r, r_{m}\right)=\frac{r^{\gamma} r_{m}^{\gamma}}{r^{2}\left(r^{\gamma}+r_{m}^{\gamma}\right)^{2}}>r_{m}^{-2} \exp (-3(s+2) \gamma-2 s-6) \quad\left(\gamma=\frac{\pi}{\beta}\right)
$$


Using (2.19) in (2.17) and (2.18) we find

$$
\begin{gathered}
\int_{r_{m} e^{-s-2}}^{r_{m} e^{s+2}} r m\left(r, f ;\left[\omega_{m}+\beta, \omega_{m}+2 \pi-\beta\right]\right) d r \leqq \eta_{m} r_{m}^{2} T\left(r_{m}\right), \\
\int_{r_{m} e^{-s-2}}^{r_{m} e^{s+2}} r m\left(r, 1 / f ;\left[\omega_{m}-\beta, \omega_{m}+\beta\right]\right) d r \leqq \eta_{m} r_{m}^{2} T\left(r_{m}\right) .
\end{gathered}
$$

By Lemma 2.1 we deduce (2.12) from (2.14) and (2.20). Similarly (2.15) and (2.21) imply (2.13). The proof of Lemma 2.2 is now complete.

3. An upper bound for $\varphi_{m}(z)$ on lines $\theta=$ constant. Apply Lemma 11.2 [A] to $f\left(z e^{i\left(\eta+\omega_{m}\right)}\right)$, with given $\eta(0<\eta<\beta)$. The sector $\mathscr{D}_{m}$ defined in (11.23 [A]) has to be replaced by

We thus obtain

$$
\Gamma_{m}=\left\{z: r_{m}^{\prime}<r \leqq r_{m}^{\prime \prime}, \omega_{m}+\eta-\beta \leqq \theta \leqq \omega_{m}+\eta+\beta\right\} .
$$

$$
\begin{aligned}
& \log \left|f\left(r e^{i\left(\eta+\omega_{m}\right)}\right)\right| \leqq \gamma^{2} \int_{r_{m}^{\prime}}^{r_{m}^{\prime \prime}} m\left(t, f ;\left[\omega_{m}+\eta-\beta, \omega_{m}+\eta+\beta\right]\right) X(t, r) d t / t \\
&+\sum_{b_{j} \in \Gamma_{m}} H\left(b_{j} e^{-i\left(\eta+\omega_{m}\right)}, r\right)+\tilde{S}_{m} \quad(\gamma=\pi / \beta) .
\end{aligned}
$$

By (11.25[A]), if $r \in I_{m}(s)$,

$$
|\tilde{S}| \leqq 56 \gamma e^{s \gamma}\left\{\left(r_{m}^{\prime} / r_{m}\right)^{\gamma} T\left(2 r_{m}^{\prime}\right)+\left(r_{m} / r_{m}^{\prime \prime}\right)^{\gamma} T\left(2 r_{m}^{\prime \prime}\right)\right\}+K\left(s+\log r_{m}\right)<\eta_{m} T\left(r_{m}\right) .
$$

We next add $\log \left|P\left(r e^{i\left(\eta+\omega_{m}\right)}\right)\right|$ to both sides of the inequality (3.1) and find

$$
\varphi_{m}\left(r e^{i\left(\eta+\omega_{m}\right)}\right) \leqq \gamma^{2} \int_{r_{m}^{\prime}}^{r_{m}^{\prime \prime}} m(t, f) X(t, r) d t / t+E_{m}+\eta_{m} T\left(r_{m}\right),
$$

$$
E_{m}=\sum_{b_{j} \in \Gamma_{m}} H\left(b_{j} e^{-i\left(\eta+\omega_{m}\right)}, r\right)+\log \left|P_{m}\left(r e^{i\left(\eta+\omega_{m}\right)}\right)\right| .
$$

Now take into account the obvious cancellation between some of the logarithmic poles introduced by the sum in the right-hand side of (3.3) and some of the poles introduced by $\log \left|P_{m}\right|$.

Let $\tilde{\mathscr{P}}_{m}$ be the complement of $\mathscr{P}_{m}$ in the annulus $r_{m}^{\prime}<|z| \leqq r_{m}^{\prime \prime} \quad\left(\mathscr{P}_{m}\right.$ is defined in (1.10)). No pole in $\tilde{\mathscr{P}}_{m}$ contributes a factor to $P_{m}(z)$ (for $m$ large enough). Let

$$
\mathscr{J}_{m}=\mathscr{P}_{m} \cap \Gamma_{m},
$$

and let $\tilde{\mathscr{J}}_{m}$ be the complement of $\mathscr{J}_{m}$ in $\mathscr{P}_{m}$. Then we may write

$$
E_{m}=X_{1}+X_{2}+X_{3}
$$


with

The estimate

$$
\begin{gathered}
X_{1}=\sum_{b_{j} \in \tilde{\mathscr{P}}_{m}} H\left(b_{j} e^{-i\left(\eta+\omega_{m}\right)}, r\right), \\
X_{2}=\sum_{b_{j} \in \mathscr{J}_{m}}\left\{H\left(b_{j} e^{-i\left(\eta+\omega_{m}\right)}, r\right)+\log \left|1-r e^{i\left(\eta+\omega_{m}\right)} / b_{j}\right|\right\}, \\
X_{3}=\sum_{b_{j} \in \tilde{\mathscr{J}}_{m}} \log \left|1-r e^{i\left(\eta+\omega_{m}\right)} / b_{j}\right| .
\end{gathered}
$$

$$
\begin{aligned}
X_{3} & \leqq p_{m} \log \left(1+e^{s+s_{m}}\right) \\
& \leqq p_{m}\left(s+s_{m}+1\right) \leqq \eta_{m} T\left(r_{m}\right)
\end{aligned}
$$

follows at once from (1.9).

To estimate $X_{2}$ we require

Lemma 3.1. If $\zeta=|\zeta| e^{i \theta}$ and

$$
|\theta| \leqq \pi / \gamma \quad(\gamma>1), \quad|\zeta|>0,
$$

then

Proof. The function

$$
\left|\frac{1-\zeta}{1-\zeta^{\gamma}}\right|<2
$$

$$
\frac{1-\zeta}{1-\zeta^{\gamma}} \quad\left(\zeta^{\gamma}=|\zeta|^{\gamma} e^{i \gamma \theta}\right)
$$

is single-valued and regular in the angle defined by (3.9). On the sides of the angle we have

$$
\left|1-\zeta^{\gamma}\right|=1+|\zeta|^{\nu}
$$

Consequently, the maximum modulus principle shows that

$$
\left|\frac{1-\zeta}{1-\zeta^{\gamma}}\right| \leqq \frac{1+|\zeta|}{1+|\zeta|^{\gamma}}<2
$$

throughout the angle (3.9). This proves Lemma 3.1.

Now apply (3.10) with

and

$$
\zeta=r / b_{j} e^{-i\left(\eta+\omega_{m}\right)} \quad(r>0)
$$

$$
\left|\arg b_{j}-\left(\eta+\omega_{m}\right)\right| \leqq \beta=\pi / \gamma .
$$

We thus find, for $b_{j} \in \mathscr{J}_{m}$ consequently

$$
\log \left|1-r e^{i\left(\eta+\omega_{m}\right)} / b_{j}\right|+\log \frac{\left|b_{j}\right|^{\gamma}}{\left|b_{j}^{\gamma} \exp \left\{-i \gamma\left(\eta+\omega_{m}\right)\right\}-r^{\gamma}\right|}<\log 2 ;
$$

$$
\log \left|1-r e^{i\left(\eta+\omega_{m}\right)} / b_{j}\right|+H\left(b_{j} e^{-i\left(\eta+\omega_{m}\right)}, r\right)<\log 2+\log \left(\left(\left|b_{j}\right|^{\gamma}+r^{\gamma}\right) /\left|b_{j}\right|^{\gamma}\right),
$$

and, in view of (1.9),

$$
X_{2}<p_{m}\left\{\log 2+\log \left(1+e^{\gamma\left(s+s_{m}\right)}\right)\right\}=\eta_{m} T\left(r_{m}\right) .
$$


We still have to estimate $X_{1}$. We may suppose that $m$ is so large that

$$
(1 / 2) \eta>\tilde{\eta}_{m} \text {. }
$$

( $\tilde{\eta}_{m}$ is the number occurring in the definition of $\mathscr{P}_{m}$ in $(1.10)$.) Then, for $b \in \tilde{\mathscr{P}}_{m}$, we find

$$
\left|\left\{b_{j} e^{-i\left(\eta+\omega_{m}\right)}\right\}^{\gamma}-r^{\gamma}\right| \geqq\left(\left|b_{j}\right|^{\gamma}+r^{\gamma}\right) \sin \gamma \eta / 4
$$

We now prove

Lemma 3.2. If $\varepsilon>0$ and $0<\eta<\beta$, then

$$
E_{m}<\left(r / r_{m}\right)^{\mu} T\left(r_{m}\right)\{\pi \mu \cos (\beta \mu) \tan (\beta \mu / 2)+\varepsilon\},
$$

provided $r \in I_{m}(s), m>m_{0}$.

Proof. Let $\sigma(0<\sigma<1)$ be a parameter to be selected later.

From Theorem 1 [A] we conclude that

$$
n\left(e^{2 \sigma} r, f\right)-n\left(e^{-\sigma} r, f\right)=\left(e^{2 \sigma \mu}-e^{-\sigma \mu}\right) T(r) \mu \cos (\beta \mu)+\eta(r) T(r) \quad\left(r \in I_{m}(s)\right) .
$$

Choose $\sigma$ so small that (3.14) implies

$$
n\left(e^{2 \sigma} r, f\right)-n\left(e^{-\sigma} r, f\right)<\{\varepsilon /(4 \log (1 / \sin (\eta / 4)))\} T(r) \quad\left(r \in I_{m}(s), m>m_{0}\right) .
$$

We next appeal to Lemma 13.1 [A] and take $x=u=r$; this yields

$$
\begin{aligned}
& \sum_{r_{m}^{\prime}<\left|b_{j}\right| \leqq r^{-\sigma}} H\left(\left|b_{j}\right|, r\right)+\sum_{r e^{2 \sigma}<\left|b_{j}\right| \leqq r_{m}^{\prime \prime}} H\left(\left|b_{j}\right|, r\right) \\
& \leqq\left(r / r_{m}\right)^{\mu} T\left(r_{m}\right)\left\{\pi \mu \cos (\beta \mu) \tan (\beta \mu / 2)+\eta_{m}\right\} .
\end{aligned}
$$

By (3.12) and (3.15) we find

$$
\begin{aligned}
\sum_{\substack{b_{j} \in \tilde{\mathscr{P}}_{m} \\
e^{-\sigma} r<\left|b_{j}\right| \leqq e^{2 \sigma} \boldsymbol{r}}} H\left(b_{j} e^{-i\left(\eta+\omega_{m}\right)}, r\right) & \leqq\left\{n\left(e^{2 \sigma} r, f\right)-n\left(e^{-\sigma} r, f\right)\right\} \log (1 / \sin (\eta / 4)) \\
& \leqq(\varepsilon / 4) T(r) \leqq(\varepsilon / 3) T\left(r_{m}\right)\left(r / r_{m}\right)^{\mu},
\end{aligned}
$$

and, in view of (3.16), we obtain

$$
X_{1}<\left(r / r_{m}\right)^{\mu} T\left(r_{m}\right)\{\pi \mu \cos (\beta \mu) \tan (\beta \mu / 2)+\varepsilon / 2\}
$$

Using the above inequality, (3.11) and (3.8), in (3.4), we deduce (3.13). This completes the proof of Lemma 3.2.

Lemma 3.3. Given $\varepsilon>0, \eta(0<\eta<\beta)$ and $s>0$, we have

provided

$$
\varphi_{m}\left(r e^{i\left(\eta+\omega_{m}\right)}\right)<(\pi \mu \sin (\beta \mu)+\varepsilon)\left(r / r_{m}\right)^{\mu} T\left(r_{m}\right),
$$

$$
r \in I_{m}(s), \quad m>m_{0}(\varepsilon, \eta, s) .
$$


Proof. By (1.2), (1.3) and $r \in I_{m}(s)$, we have

and

$$
\begin{aligned}
& \gamma^{2} \int_{r_{m}^{\prime}}^{r_{m}^{\prime \prime}} m(t, f) X(t, r) d t / t=\gamma^{2} \int_{r_{m}^{\prime}}^{r_{m}^{\prime \prime}}\{T(t)-N(t, f)\} X(t, r) d t / t \\
& \leqq\left(1+\eta_{m}\right)(1-\cos \beta \mu) \gamma^{2} T\left(r_{m}\right)\left(r / r_{m}\right)^{\mu} \int_{r_{m}^{\prime}}^{r_{m}^{\prime \prime}}(t / r)^{\mu} X(t, r) d t / t,
\end{aligned}
$$

$$
\gamma^{2} \int_{r_{m}^{\prime}}^{r_{m}^{\prime \prime}}(t / r)^{\mu} X(t, r) d t / t<\gamma \int_{0}^{\infty} \frac{u^{\mu / \gamma} d u}{(1+u)^{2}}=\frac{\pi \mu}{\sin (\beta \mu)} .
$$

Using (3.17), (3.18), and (3.13) in (3.2) we obtain the desired result.

4. The behavior of $\varphi_{m}\left(r e^{i \theta}\right)$ in $\left|\theta-\omega_{m}\right|<\beta$.

Lemma 4.1. Let $\varepsilon>0, \eta(0<\eta<\min (\beta, \pi-\beta))$ and $s>0$ be given. Then, if

$$
r \in I_{m}(s), \quad \eta \leqq|\theta| \leqq \beta, \quad m>m_{0}(\varepsilon, \eta, s),
$$

we have

$$
\varphi_{m}\left(r e^{i \theta+i \omega_{m}}\right) \leqq\{\pi \mu \sin \mu(\beta-|\theta|)+\varepsilon\}\left(r / r_{m}\right)^{\mu} T\left(r_{m}\right) .
$$

Proof. We consider only the interval $\eta \leqq \theta \leqq \beta$. The proof for $-\beta \leqq \theta \leqq-\eta$ is exactly the same.

To prove the lemma we apply Lemma 2.1 [A], in a suitable sector, to the subharmonic function $\varphi_{m}(z)$.

Since we consider one such sector at a time we suppose, for simplicity of writing, that $\omega_{m}=0$. If this is not initially the case, replace $f(z)$ by $f\left(z e^{i \omega_{m}}\right)$.

We now apply Lemma $2.1[\mathrm{~A}]$ to $\varphi_{m}(z)$ with

$$
\varrho=\mu, \quad l=e^{-S} r_{m}, \quad R=e^{S} r_{m}, \quad \gamma=\pi / \beta \geqq 2 \mu ;
$$

$S>0$ and an additional parameter $\eta^{\prime}>0$ are selected as follows.

We take $\eta^{\prime}=\eta^{\prime}(\varepsilon, \eta)$ to be the largest number such that

$$
\eta^{\prime} \leqq \eta / 2, \quad \pi \mu \sin \left(\mu \eta^{\prime}\right) \leqq \varepsilon / 5,
$$

and $S=S(\varepsilon, \eta, s)$ to be the smallest number such that

$$
S \geqq s+1, \quad 6 e^{-(S-s) \mu}\left(\frac{2 e^{\mu+2}}{\sin ^{2}\left(\eta^{\prime} / 2\right)}+\frac{\varepsilon}{2}+\pi \mu\right) \leqq \frac{\varepsilon}{4} .
$$

Put

$$
\theta_{1}=\eta^{\prime}, \quad \theta_{2}=\beta+\eta^{\prime}, \quad B=\frac{2 e^{\mu+2}}{\sin ^{2}\left(\eta^{\prime} / 2\right)} r_{m}^{-\mu} T\left(r_{m}\right) .
$$

Lemma 2.2 with $s+1$ replaced by $S$ shows that, for

$$
r_{m} e^{-S} \leqq|\zeta| \leqq r_{m} e^{S},
$$


and

we have

$$
\theta_{1} \leqq \arg \zeta=\theta \leqq \theta_{2}, \quad m>m_{0}\left(\eta^{\prime}, S\right),
$$

$$
\begin{gathered}
\varphi_{m}(\zeta) \leqq B|\zeta|^{\mu}, \\
\varphi_{m}\left(|\zeta| e^{\left.i \theta_{2}\right)} \leqq \eta_{m} T\left(r_{m}\right) \leqq(\varepsilon / 4) e^{-S \mu} \sin (\beta \mu) T\left(r_{m}\right)\right. \\
\leqq \varepsilon / 4 \sin (\beta \mu) r_{m}^{-\mu} T\left(r_{m}\right)|\zeta|^{\mu}=h_{2}|\zeta|^{\mu} .
\end{gathered}
$$

Similarly, for $m$ large enough, (4.6) and Lemma 3.3 imply

$$
\varphi_{m}\left(|\zeta| e^{i \theta_{1}}\right) \leqq(\pi \mu+\varepsilon / 4) \sin (\beta \mu) r_{m}^{-\mu} T\left(r_{m}\right)|\zeta|^{\mu}=h_{1}|\zeta|^{\mu} .
$$

In view of (4.5), (13.3 [A]) and (13.4 [A]) we also have

$$
\theta_{2}-\theta_{1}=\beta=\pi / \gamma, \quad \gamma>1, \quad \gamma-\mu \geqq \mu>0 .
$$

The relations (4.7), (4.8), (4.9) and (4.10) enable us to apply Lemma 2.1 [A]. We thus find that, under the restrictions (4.1), (2.7 [A]) yields

$$
\begin{aligned}
\varphi_{m}(z) & \leqq\left\{r / r_{m}\right\}^{\mu} T\left(r_{m}\right)\left\{(\pi \mu+\varepsilon / 4) \sin \mu\left(\beta+\eta^{\prime}-\theta\right)+(\varepsilon / 4) \sin \mu\left(\theta-\eta^{\prime}\right)\right. \\
& \left.+6 e^{-(S-s) \mu}\left(\frac{2 e^{\mu+2}}{\sin ^{2}\left(\eta^{\prime} / 2\right)}+\frac{\varepsilon}{2}+\pi \mu\right)\right\} .
\end{aligned}
$$

Taking (4.3) and (4.4) into account we obtain (4.2).

Our next lemma is an immediate consequence of a result which we have used repeatedly [2; Lemma III].

Lemma 4.2. Let $\varepsilon>0$ and $s>0$ be given. It is possible to determine $\delta=\delta(\varepsilon, s)>0$ such that, if $E$ is a measurable set and if

then

$$
|E|<\delta, \quad r \in I_{m}(s), \quad m>m_{0}(\varepsilon, s),
$$

$$
\begin{aligned}
& \frac{1}{2 \pi} \int_{E}\left\{|\log | f\left(r e^{i \theta}\right)||+|\log | P_{m}\left(r e^{i \theta}\right)||\right. \\
& \left.\quad+|\log | Z_{m}\left(r e^{i \theta}\right)||\right\} d \theta<\varepsilon T\left(r_{m}\right) .
\end{aligned}
$$

Proof. By [2; 322, Lemma III] (applied to both $f$ and $1 / f$ ) and (1.2)

$$
\begin{gathered}
\frac{1}{2 \pi} \int_{E}|\log | f\left(r e^{i \theta}\right)|| d \theta \leqq 45\left(2 r / r_{m}\right)^{\mu} \delta\left(1+\log ^{+}(1 / \delta)\right) T\left(r_{m}\right) \\
\left(r \in I_{m}(s), m>m_{0}(\varepsilon, s)\right) .
\end{gathered}
$$

The lemma now follows from (4.12), (1.20) and the remark that

$$
\delta(1+\log (1 / \delta)) \rightarrow 0 \quad(\delta \rightarrow 0+) .
$$

In the following lemma we confine our attention to values of $\theta$ in $\left[\omega_{m}, \pi+\omega_{m}\right]$; the interval $\left[-\pi+\omega_{m}, \omega_{m}\right]$ is treated by symmetry. 
Lemma 4.3. The function

$$
\Omega_{m}(z)=\psi_{m}(z)-\left(|z| / r_{m}\right)^{\mu} T\left(r_{m}\right) \pi \mu \sin \mu\left(\beta-\theta+\omega_{m}\right)
$$

is harmonic in $\mathscr{D}_{m}$ (defined by (1.15)).

Given $s>0$ and $\varepsilon>0$, we have

$$
\frac{1}{2 \pi} \int_{\omega_{m}}^{\beta+\omega_{m}}\left|\Omega_{m}\left(r e^{i \theta}\right)\right| d \theta<\varepsilon T\left(r_{m}\right),
$$

provided

$$
r \in I_{m}(s+1), \quad m>m_{0}(\varepsilon, s) .
$$

Proof: The harmonicity of $\Omega_{m}(z)$ follows from the harmonicity of $\psi_{m}(z)$ (defined in (1.14)) and the remark that

$$
-|z|^{\mu} \sin \mu(\beta-\theta)=\operatorname{Im}\left\{z^{\mu} e^{-i \mu \beta}\right\} .
$$

[For simplicity of writing we assume that $\omega_{m}=0$.]

Using Lemma 4.2, we first determine $\eta=\eta(\varepsilon, s)>0$ such that under the conditions

$$
\begin{gathered}
r \in I_{m}(s+1), \quad m>m_{0}(\varepsilon, s), \quad|E| \leqq 5 \eta, \\
\frac{1}{2 \pi} \int_{E}\left\{|\log | f\left(r e^{i \theta}\right)||+|\log | P_{m}\left(r e^{i \theta}\right)||\right. \\
\left.+|\log | Z_{m}\left(r e^{i \theta}\right)||+\pi \mu e^{(s+1) \mu} T\left(r_{m}\right)\right\} d \theta<\left(\varepsilon e^{-(s+1) \mu} / 14\right) T\left(r_{m}\right),
\end{gathered}
$$

where $E$ is a measurable set of arguments.

From Lemma 4.1, (1.20) and (4.16) we readily see that

$$
\frac{1}{2 \pi} \int_{0}^{\beta} \Omega_{m}^{+}\left(r e^{i \theta}\right) d \theta \leqq(\varepsilon / 6) T\left(r_{m}\right) \quad\left(r \in I_{m}(s+1), m>m_{0}\right)
$$

as well as

$$
m(r, f ;[-\beta,-\eta]) \leqq\left(r / r_{m}\right)^{\mu} T\left(r_{m}\right)\left\{(1-\cos \beta \mu) / 2+\varepsilon e^{-(s+1) \mu} / 14\right\}
$$

We next show that

$$
\left(r \in I_{m}(s+1), m>m_{0}\right) \text {. }
$$

$$
\frac{1}{2 \pi} \int_{\eta}^{\beta-\eta} \Omega_{m}\left(r e^{i \theta}\right) d \theta \geqq-(\varepsilon / 6) T\left(r_{m}\right) \quad\left(r \in I_{m}(s+1), m>m_{0}\right) .
$$

If this were not true, there would be arbitrarily large values of $\varrho=\varrho_{m}$ such that

$$
\frac{1}{2 \pi} \int_{\eta}^{\beta-\eta} \Omega_{m}\left(\varrho e^{i \theta}\right) d \theta<-(\varepsilon / 6) T\left(r_{m}\right) \quad\left(\varrho=\varrho_{m} \in I_{m}(s+1)\right) .
$$

By the definition of $\Omega_{m}(z)$ and (1.20), the above inequality implies

$$
\begin{aligned}
& \frac{1}{2 \pi} \int_{\eta}^{\beta-\eta} \log \left|f\left(\varrho e^{i \theta}\right)\right| d \theta<\frac{1}{2 \pi} \int_{\eta}^{\beta-\eta} \log \left|1 / f\left(\varrho e^{i \theta}\right)\right| d \theta \\
& +\left(\varrho / r_{m}\right)^{\mu} T\left(r_{m}\right)(1-\cos \beta \mu) / 2+\left(-(\varepsilon / 6)+\eta_{m}\right) T\left(r_{m}\right) .
\end{aligned}
$$


Now

$$
\stackrel{+}{\log }|1 / f| \leqq\left\{-\psi_{m}\right\}^{+}+\stackrel{+}{\log }\left|P_{m}(z)\right|+\stackrel{+}{\log }\left|1 / Z_{m}(z)\right|,
$$

and hence, by (1.20) and (2.13), (4.21) yields

$$
m(\varrho, f ;[\eta, \beta-\eta]) \leqq\left(\varrho / r_{m}\right)^{\mu}\left\{(1-\cos \beta \mu) / 2-(\varepsilon / 6) e^{-(s+1) \mu}+\eta_{m}\right\} T\left(r_{m}\right) \quad\left(m>m_{0}\right) .
$$

Using (2.12) instead of (2.13), we see that

$$
m(\varrho, f ;[\beta+\eta, 2 \pi-\beta-\eta]) \leqq \eta_{m} T\left(r_{m}\right) .
$$

We now choose in (4.16)

$$
E=[-\eta, \eta] \cup[\beta-\eta, \beta+\eta] \cup[2 \pi-\beta-\eta, 2 \pi-\beta]
$$

and combine the resulting relation with (4.18) (with $r$ replaced by $\varrho$ ), (4.22) and (4.23). This leads to

$$
\begin{gathered}
m\left(\varrho_{m}, f\right) \leqq\left(\varrho_{m} / r_{m}\right)^{\mu} T\left(r_{m}\right)\left\{1-\cos \beta \mu-(\varepsilon / 43) e^{-(s+1) \mu}\right\} \quad\left(m>m_{0}\right) \\
\liminf _{r \rightarrow \infty} \frac{m(r, f)}{T(r)}<(1-\cos \beta \mu) \quad\left(r \in I_{m}(s+1)\right) .
\end{gathered}
$$

The latter inequality rewritten as

$$
\limsup _{r \rightarrow \infty} \frac{N(r, f)}{T(r)}>\cos \beta \mu \quad\left(r \in I_{m}(s+1)\right),
$$

contradicts (16[A]) and hence shows the impossibility of (4.20). We have thus proved (4.19).

Using (4.16) once more, we find

$$
\frac{1}{2 \pi} \int_{0}^{\beta} \Omega_{m}\left(r e^{i \theta}\right) d \theta \geqq-(\varepsilon / 3) T\left(r_{m}\right) \quad\left(r \in I_{m}(s+1), m>m_{0}\right)
$$

which, combined with (4.17), yields

$$
(\varepsilon / 2) T\left(r_{m}\right) \geqq \frac{1}{2 \pi} \int_{0}^{\beta}\left(\Omega_{m}^{+}\left(r e^{i \theta}\right)+\left\{-\Omega_{m}\left(r e^{i \theta}\right)\right\}^{+}\right) d \theta \quad\left(r \in I_{m}(s+1), m>m_{0}\right),
$$

and (4.14) follows. The proof of Lemma 4.3 is now complete.

5. Proof of Theorem 4. Part II of Theorem 4 is already part of the assertions of Theorem $3[\mathrm{~A}]$.

Consider now the given quantities $s, \varepsilon, \eta$ which appear in Theorem 4 . The definition of $\varphi_{m}$ and (2.12) yield

$$
\log \left|f\left(r e^{i \theta}\right)\right|<\log \left|1 / P_{m}\left(r e^{i \theta}\right)\right|+\eta_{m} T\left(r_{m}\right) \quad\left(\beta+\eta \leqq|\theta| \leqq \pi, r \in I_{m}(s)\right) .
$$

Taking into account (1.21) and (1.2), we see that (5.1) implies (2). 
To prove (3) we note that, by Lemma 4.3

$$
\frac{1}{2 \pi} \int_{\omega_{m}+\eta / 2}^{\beta+\omega_{m}-\eta / 2}\left|\Omega_{m}\left(r e^{i \theta}\right)\right| d \theta \leqq \varepsilon^{\prime} T\left(r_{m}\right) \quad\left(r \in I_{m}(s+1), m>m_{0}\left(\varepsilon^{\prime}, s\right)\right),
$$

where we have chosen

$$
\varepsilon^{\prime}=(\varepsilon / 2) \sin ^{2}(\eta / 2) e^{-(4+\mu) s-2} .
$$

Using (5.2) in Lemma 2.1 we obtain

$$
\left|\Omega_{m}(z)\right| \leqq(\varepsilon / 2) e^{-s \mu} T\left(r_{m}\right) \quad\left(\omega_{m}+\eta \leqq \theta \leqq \omega_{m}+\beta-\eta, r \in I_{m}(s)\right)
$$

and hence, by (4.13), (1.13) and (1.14)

$$
\begin{gathered}
|\log | f(z)\left|-\left(r / r_{m}\right)^{\mu} T\left(r_{m}\right) \pi \mu \sin \mu\left(\beta-\theta+\omega_{m}\right)\right| \leqq(\varepsilon / 2) e^{-s \mu} T\left(r_{m}\right) \\
+|\log | P_{m}(z)||+|\log | Z_{m}(z)|| .
\end{gathered}
$$

Taking (1.19) and (1.21) into account, we readily complete the proof of (3) and, consequently, of Theorem 4.

6. Proof of Carleman's identity for a sector. We may consider

$$
G(z)=g(z) \prod_{b \in D}(1-z / b),
$$

and treat separately $G(z)$ and the polynomial in (6.1). Then, as in all such identities, the final result is obtained by subtracting the formula for the polynomial from the formula for $G$.

We confine our attention to the special case $c=1$; using an elementary change of variable we then obtain the general case.

The preceding simplifications enable us to assume that $g(z)$ is regular in the sector

$$
D_{1}: \operatorname{Re} z \geqq 0, \quad l \leqq|z| \leqq R \quad\left(l=t e^{-\sigma}, R=t e^{\sigma}\right) ;
$$

the zeros of $g(z)$ in $D_{1}$ are $a_{j}=\left|a_{j}\right| e^{i \omega_{j}}(j=1,2, \ldots, n)$.

Consider the auxiliary function

$$
U(z)=g(z) \bar{g}\left(t^{2} z^{-1}\right)
$$

which is regular in

$$
D_{2}: \operatorname{Re} z \geqq 0, \quad t \leqq|z| \leqq R .
$$

The boundary $\partial D_{2}$ will be used as contour of integration; taken in the positive sense, it will be denoted by $\mathscr{C}$.

We assume first that $f(z)$ has no zeros on $\partial D_{1}$ and no zeros of modulus $t$. At this stage of the proof these restrictions are essential.

The collection of zeros of $U(z)$ in $D_{2}$ is easily described; it is formed by

(i) all those points $a_{j}$ such that $t<\left|a_{j}\right|<R$, and

(ii) all the points $t^{2} / \bar{a}_{j}$ such that $l<\left|a_{j}\right|<t$. 
We now follow, step by step, some simple proof of Carleman's theorem; for instance the one given by Titchmarsh [4; pp. 130-131].

We consider the integral

$$
\frac{1}{2 \pi i} \int_{\mathscr{C}} \log U(z)\left(1 / z^{2}+1 / R^{2}\right) d z=I,
$$

and start the integration at $z=i t$ with the real determination of the logarithm. There is such a determination since

$$
\log U\left(t e^{i \theta}\right)=\log \left|g\left(t e^{i \theta}\right)\right|^{2} \quad(-\pi / 2 \leqq \theta \leqq \pi / 2) .
$$

Using this relation in (6.3) we see that the contribution of the portion of the integral corresponding to the semicircle of radius $t$ is

$$
-\frac{1}{\pi} \int_{-\pi / 2}^{\pi / 2} \log \left|g\left(t e^{i \theta}\right)\right|\left\{e^{-i \theta} / t+t e^{i \theta} / R^{2}\right\} d \theta
$$

For the large semicircle we obtain

$$
\frac{1}{\pi R} \int_{-\pi / 2}^{\pi / 2} \log \left\{g\left(R e^{i \theta}\right) \bar{g}\left(l e^{-i \theta}\right)\right\} \cos \theta d \theta .
$$

For the two intervals of the imaginary axis we find

$$
\frac{1}{2 \pi} \int_{t}^{R} \log \left\{g(i y) g(-i y) \bar{g}\left(i t^{2} / y\right) \bar{g}\left(-i t^{2} / y\right)\right\}\left(1 / y^{2}-1 / R^{2}\right) d y .
$$

Using (6.4), (6.5) and (6.6) in the evaluation of $I$, we are led to

$$
\begin{aligned}
\operatorname{Re} I= & -\left(1 / t+t / R^{2}\right) \frac{1}{\pi} \int_{-\pi / 2}^{\pi / 2} \log \left|g\left(t e^{i \theta}\right)\right| \cos \theta d \theta \\
& +\frac{1}{\pi R} \int_{-\pi / 2}^{\pi / 2} \log \left|g\left(R e^{i \theta}\right) g\left(l e^{i \theta}\right)\right| \cos \theta d \theta \\
& +\frac{1}{2 \pi} \int_{l}^{t} \log |g(i y) g(-i y)|\left(1 / t^{2}-t^{2} / y^{2} R^{2}\right) d y \\
& +\frac{1}{2 \pi} \int_{t}^{R} \log |g(i y) g(-i y)|\left(1 / y^{2}-1 / R^{2}\right) d y .
\end{aligned}
$$

An integration by parts yields another evaluation of I:

$$
2 \pi i I=\left\{\left(-1 / z+z / R^{2}\right) \log U(z)\right\}_{\mathscr{C}}+\int_{\mathscr{C}}\left\{\left(1 / z-z / R^{2}\right)\left(U^{\prime}(z) / U(z)\right) d z\right\} .
$$

By the argument principle, the integrated term of the right-hand side of (6.8) is a 
real quantity and hence we deduce from (6.8):

$$
\begin{aligned}
\operatorname{Re} I & =\operatorname{Re}\left\{\frac{1}{2 \pi i} \int_{\mathscr{C}}\left(1 / z-z / R^{2}\right)\left(U^{\prime}(z) / U(z)\right) d z\right\} \\
& =\operatorname{Re}\left\{\sum_{l<\left|a_{j}\right|<t}\left(\frac{\bar{a}_{j}}{t^{2}}-\frac{t^{2}}{\bar{a}_{j} R^{2}}\right)+\sum_{t<\left|a_{j}\right|<R}\left(\frac{1}{a_{j}}-\frac{a_{j}}{R^{2}}\right)\right\} .
\end{aligned}
$$

The last expression has been obtained by an elementary evaluation of residues.

From (6.7) and (6.9) we deduce (16) in the special case $c=1$. If $g(z)$ has zeros on the imaginary axis (but not on the three circles $|z|=l,|z|=t,|z|=R$ ) we may following Titchmarsh, make small indentations round these zeros and proceed to the limit. It is immediately verified that the validity of (16) is not affected in this case.

If $g(z)$ has zeros on the circular arcs of radii $l, t, R$, we apply (16) with $l, t, R$ replaced, respectively, by

$$
l^{\prime}=l(1-\varepsilon), \quad t^{\prime}=t(1-\varepsilon), \quad R^{\prime}=R(1-\varepsilon) \quad(0<\varepsilon<1),
$$

and then let $\varepsilon \rightarrow 0+$. The elementary, continuity arguments which justify this step offer no difficulties and will be left to the reader.

To pass from the case $c=1$ to the general one, we apply the special case of the identity to the function

$$
W(\zeta)=g\left(\zeta^{1 / c}\right), \quad z=\zeta^{1 / c}
$$

To obtain (16) we eliminate from the final form of the identity the auxiliary variable $\zeta$ and the auxiliary function $W(\zeta)$.

7. Consequences of Carleman's identity for a sector. Our identity (16) immediately yields simple and precise inequalities for the logarithmic mean denoted below by $H(t)$. We prove

Lemma 7.1. Let $g(z)$ be regular and let

$$
|g(z)| \leqq M \quad(M>0)
$$

throughout the sector

Put

$$
v e^{-2 \sigma} \leqq|z| \leqq v e^{2 \sigma}, \quad-\pi / 2 c \leqq \theta=\arg z \leqq \pi / 2 c \quad(v>0, \sigma>0, c>1 / 2) .
$$

I. Then

$$
H(t)=\frac{1}{2 \pi} \int_{-\pi / 2 c}^{\pi / 2 c} \log \left|g\left(t e^{i \theta}\right)\right| \cos (c \theta) d \theta
$$

$$
\begin{gathered}
2(t / v)^{c} H(v)+\left(1-2(t / v)^{c}\right)(\log M) / \pi c \leqq H(t) \\
\leqq(1 / 2)(v / t)^{c} H(v)+\left(1-(1 / 2)(v / t)^{c}\right)(\log M) / \pi c \quad\left(v \leqq t \leqq v e^{\sigma}\right), \\
2(v / t)^{c} H(v)+\left(1-2(v / t)^{c}\right)(\log M) / \pi c \leqq H(t) \\
\leqq(1 / 2)(t / v)^{c} H(v)+\left(1-(1 / 2)(t / v)^{c}(\log M) / \pi c \quad\left(v e^{-\sigma} \leqq t \leqq v\right) .\right.
\end{gathered}
$$


II. There are, in the sector

$$
\Delta^{*}=\left\{z: v e^{-\sigma} \leqq|z| \leqq v e^{\sigma},-\pi / 2 c_{1} \leqq \theta \leqq \pi / 2 c_{1}\right\} \quad\left(c_{1}>c\right),
$$

no more than

$$
n^{*}\left[\leqq 4 c e^{\sigma c} / \cos \left(\pi c / 2 c_{1}\right)\left(1-e^{-2 \sigma c}\right)\right]\{(\log M) / \pi c-H(v)\},
$$

zeros of $g(z)$.

Proof of I. We establish (7.2) in the special case $M=1$, for which

$$
H(t) \leqq 0 \quad\left(v e^{-2 \sigma} \leqq t \leqq v e^{2 \sigma}\right) .
$$

The general form of (7.2) follows at once from the special one. Let

$$
s=\log (t / v) \geqq 0 .
$$

By (16), (7.1), (7.5), (7.6) and the remark that $\sum_{a} \eta(a) \geqq 0$, we find

as well as

$$
2 e^{s c} H(v) \leqq H\left(v e^{s}\right)=H(t) \quad(0 \leqq s \leqq 2 \sigma),
$$

Hence

$$
2 e^{s c} H\left(v e^{s}\right) \leqq H(v) \quad(0 \leqq s \leqq \sigma) .
$$

$$
2(t / v)^{c} H(v) \leqq H(t) \leqq(1 / 2)(v / t)^{c} H(v) \quad\left(v \leqq t \leqq v e^{\sigma}\right),
$$

which is (7.2) in the special case $M=1$.

The same arguments, with insignificant modifications, yield (7.3) and thus complete the proof of assertion I.

Proof of II. We start from (16) with $g(z)$ replaced by $g(z) / M$; $t$ is to be replaced by $v$ and $\sigma$ by $2 \sigma$. Then

$$
\sum_{a \in \Delta^{*}} \eta(a) \leqq \Sigma_{a} \eta(a) \leqq 2 c e^{2 \sigma c} / \pi \int_{-\pi / 2 c}^{\pi / 2 c} \log \left|M / g\left(v e^{i \theta}\right)\right| \cos (c \theta) d \theta,
$$

where

$$
\eta(a) \geqq\left(e^{\sigma c}-e^{-\sigma c}\right) \cos \left(\pi c / 2 c_{1}\right) \quad\left(a \in \Delta^{*}\right) .
$$

The bound in (7.4) follows at once from (7.7) and (7.8).

Lemma 7.2. Let $g(z)$ be meromorphic in the whole $z$-plane.

I. Assume that

(i) $g(z)$ is regular in the sector

$$
\begin{gathered}
\Delta=\left\{z: v e^{-2 \sigma-s-1} \leqq r \leqq v e^{2 \sigma+s+1},-\pi / 2 c \leqq \theta \leqq \pi / 2 c\right\} \\
(2 \sigma>s+1>1, v>0, c>1 / 2) ;
\end{gathered}
$$

(ii) for some given $M \geqq 1$ and $B>0$, the following inequalities are satisfied

$$
\begin{gathered}
|g(z)| \leqq M \quad(z \in \Delta), \\
m(r, 1 / g) \leqq B e^{(-\sigma+s+1) \mu} \quad\left(v e^{-\sigma-s-1} \leqq r \leqq v e^{-\sigma+s+1}\right) .
\end{gathered}
$$


Then, if

$$
v e^{-s-1} \leqq x \leqq v e^{s+1},
$$

and if $H$ is defined as in Lemma 7.1, we have

$$
-H(x) \leqq 2 B e^{\sigma(c-\mu)+(s+1) \mu}+2 e^{\sigma c} \log M .
$$

II. $g(z)$ has no more than

$$
n^{\prime}<\frac{8 e^{(s+2) c}}{\cos \left(\pi c / 2 c_{1}\right)}\left(3 e^{\sigma c} \log M+2 B e^{\sigma(c-\mu)+(s+1) \mu}\right)
$$

zeros in the sector

$$
\Delta^{\prime}=\left\{z: e^{-s-1} v \leqq r \leqq e^{s+1} v,-\pi / 2 c_{1} \leqq \theta \leqq \pi / 2 c_{1}\right\} \quad\left(c_{1}>c\right) .
$$

Proof of I. The definition of $\Delta$ and (7.11) enable us to use (7.3) with $t$ replaced by $x e^{-\sigma}$ and $v$ replaced by $x$; this yields

$$
\begin{gathered}
H\left(x e^{-\sigma}\right) \leqq(1 / 2) e^{-\sigma c} H(x)+(\log M) / \pi c \\
-H(x) \leqq 2 e^{\sigma c}\left(\frac{1}{2 \pi} \int_{-\pi / 2 c}^{\pi / 2 c}+\log \left|1 / g\left(x e^{-\sigma} e^{i \theta}\right)\right| d \theta\right)+2 e^{\sigma c} \log M .
\end{gathered}
$$

Using (7.10) we obtain (7.12).

Proof of II. We obtain (7.13) by using assertion II of Lemma 7.1 and (7.12) with $x=v$.

8. The two behaviors of $\varphi_{m}\left(r e^{i \theta}\right)$ in $\beta<\left|\theta-\omega_{m}\right| \leqq \pi$. Let

$$
0<\eta<(1 / 2) \min (\beta, \pi-\beta)
$$

Three auxiliary quantities $c, c_{1}$ and $\xi$ are defined:

$$
\pi-\beta-\eta / 3=\pi / 2 c, \quad \pi-\beta-2 \eta / 3=\pi / 2 c_{1}, \quad \xi=\cos \left(\pi c / 2 c_{1}\right) .
$$

If the condition (5) is satisfied we impose on $\eta$ the additional restriction

$$
\eta<(1 / 2)(\pi-\beta-\pi / 2 \mu)
$$

Hence we always have

$$
1 / 2<c<c_{1}, \quad 0<\xi<1
$$

if (5) is satisfied, we also have

$$
c<\mu \text {. }
$$

Observe that (1.13) may be rewritten as

$$
\varphi_{m}(z)=\log \left|g_{m}(z)\right|, \quad g_{m}(z)=f(z) P_{m}(z) .
$$

The function $g_{m}(z)$, meromorphic in the whole plane, satisfies in view of $(1.20)$

$$
m\left(r, 1 / g_{m}\right) \leqq m(r, 1 / f)+m\left(r, 1 / P_{m}\right) \leqq T(r)+\eta_{m} T\left(r_{m}\right) \quad\left(r \leqq r_{m} e^{s_{m}}\right) .
$$


Once more we simplify the notation by taking $\omega_{m}=0$ and examine

by $(2.12)$

$$
\limsup _{m \rightarrow \infty} \frac{1}{2 \pi T\left(r_{m}\right)} \int_{\beta+\eta / 3}^{2 \pi-\beta-\eta / 3} \varphi_{m}\left(r_{m} e^{i \theta}\right) \cos (c(\theta-\pi)) d \theta=\varkappa
$$

and there are exactly two possibilities

$$
\liminf _{m \rightarrow \infty} \frac{1}{2 \pi T\left(r_{m}\right)} \int_{\beta+\eta / 3}^{2 \pi-\beta-\eta / 3} \varphi_{m}\left(r_{m} e^{i \theta}\right) \cos (c(\theta-\pi)) d \theta<0
$$

and

$$
\lim _{m \rightarrow \infty} \frac{1}{2 \pi T\left(r_{m}\right)} \int_{\beta+\eta / 3}^{2 \pi-\beta-\eta / 3} \varphi_{m}\left(r_{m} e^{i \theta}\right) \cos (c(\theta-\pi)) d \theta \doteq 0 .
$$

9. Study of the case (A) and proof of part of Theorem 6. From (A) we deduce the existence of a constant $K_{0}=K_{0}(\eta)>0$ and of an infinite subsequence $\mathscr{M}$ of the positive integers such that

$$
\frac{1}{2 \pi} \int_{\beta+\eta / 3}^{2 \pi-\beta-\eta / 3} \varphi_{m}\left(r_{m} e^{i \theta}\right) \cos (c(\theta-\pi)) d \theta<-2 K_{0} T\left(r_{m}\right) \quad(m \in \mathscr{M}) .
$$

Let $s>0$ be given and use (2.12) to determine a sequence $\left\{\tilde{\eta}_{m}\right\}_{m} \quad\left(\tilde{\eta}_{m}>0, \tilde{\eta}_{m} \rightarrow 0\right)$ such that

$$
\begin{gathered}
\tilde{\varphi}_{m}\left(r e^{i \theta}\right)=\varphi_{m}\left(r e^{i \theta}\right)-\tilde{\eta}_{m} T\left(r_{m}\right) \leqq 0 \\
\left(r \in I_{m}(2(s+1)), \beta+\eta / 3 \leqq \theta \leqq 2 \pi-\beta-\eta / 3\right) .
\end{gathered}
$$

In view of (9.1) and (9.2), Lemma 7.1 is applicable to the function

$$
g_{m}(z) e^{-\tilde{n}_{m} T\left(r_{m}\right)} \text {. }
$$

Hence, using (7.2) and (7.3), we find

$$
\begin{gathered}
\frac{1}{2 \pi} \int_{\beta+\eta / 3}^{2 \pi-\beta-\eta / 3} \tilde{\varphi}_{m}\left(r e^{i \theta}\right) d \theta \leqq \frac{1}{2 \pi} \int_{\beta+\eta / 3}^{2 \pi-\beta-\eta / 3} \tilde{\varphi}_{m}\left(r e^{i \theta}\right) \cos (c(\theta-\pi)) d \theta \\
\leqq(1 / 2) e^{-c(s+1)} \frac{1}{2 \pi} \int_{\beta+\eta / 3}^{2 \pi-\beta-\eta / 3} \tilde{\varphi}_{m}\left(r_{m} e^{i \theta}\right) \cos (c(\theta-\pi)) d \theta<-K_{0} e^{-c(s+1)} T\left(r_{m}\right) \\
\left(m \in \mathscr{M}, r \in I_{m}(s+1)\right) .
\end{gathered}
$$

To pass from the "average" in (9.3) to a punctual inequality we use a result which we have established elsewhere $[2 ; 321$, Lemma I]. We thus find

$$
\log \left|f\left(r e^{i \theta}\right) P_{m}\left(r e^{i \theta}\right)\right|-\tilde{\eta}_{m} T\left(r_{m}\right)=\tilde{\varphi}_{m}\left(r e^{i \theta}\right) \leqq-K_{1} T\left(r_{m}\right),
$$

where $K_{1}=K_{1}(s, \eta)>0$ and

$$
r \in I_{m}(s), \quad \beta+2 \eta / 3 \leqq \theta \leqq 2 \pi-\beta-2 \eta / 3, \quad m \in \mathscr{U} .
$$

It is now obvious that (9.4) and (1.21) lead to the inequality (13) of Theorem 6. 
10. Study of the case (B); proof of Theorem 6 completed. By assumption, condition (B) now holds. Hence, with $\tilde{\eta}_{m}$ and $\tilde{\varphi}_{m}$ as in (9.2), the condition may be rewritten as

$$
\frac{1}{2 \pi} \int_{\beta+\eta / 3}^{2 \pi-\beta-\eta / 3}\left|\tilde{\varphi}_{m}\left(r_{m} e^{i \theta}\right)\right| \cos (c(\theta-\pi)) d \theta=\eta_{m} T\left(r_{m}\right) .
$$

Lemma 7.1 is clearly applicable and leads from (10.1) to

$$
0 \leqq \frac{1}{2 \pi} \int_{\beta+\eta / 3}^{2 \pi-\beta-\eta / 3}\left|\tilde{\varphi}_{m}\left(r e^{i \theta}\right)\right| \cos (c(\theta-\pi)) d \theta \leqq \eta_{m} T\left(r_{m}\right) \quad\left(r \in I_{m}(s+1)\right) .
$$

Denote by $q_{m}$ the number of zeros of $f(z)$ in the sector

$$
\Delta_{m}=\left\{z: r \in I_{m}(s+1), \beta+2 \eta / 3 \leqq \theta \leqq 2 \pi-\beta-2 \eta / 3\right\} .
$$

Then, by (7.4), (9.2) and (10.1)

$$
q_{m} \leqq \eta_{m} T\left(r_{m}\right) .
$$

Let

$$
Q_{m}(z)=\prod_{a \in \Delta_{m}}(1-z / a) .\left(\text { degree } Q_{m}=q_{m}\right),
$$

where the $a$ 's denote the zeros of $f(z)$. Obviously, the function

$$
\Theta_{m}(z)=\log |f(z)|+\log \left|P_{m}(z)\right|-\log \left|Q_{m}(z)\right|
$$

is harmonic in the sector $\Delta_{m}$.

From (10.2), (10.4) and Lemma 1.1 we conclude that

$$
\frac{1}{2 \pi} \int_{\beta+2 \eta / 3}^{2 \pi-\beta-2 \eta / 3}\left|\Theta_{m}\left(r e^{i \theta}\right)\right| d \theta \leqq \eta_{m} T\left(r_{m}\right) \quad\left(r \in I_{m}(s+1)\right) .
$$

An appeal to Lemma 2.1 transforms (10.6) into the punctual inequality

$$
\left|\Theta_{m}\left(r e^{i \theta}\right)\right| \leqq \eta_{m} T\left(r_{m}\right) \quad\left(r \in I_{m}(s), \beta+\eta \leqq \theta \leqq 2 \pi-\beta-\eta\right) .
$$

Using Lemma 1.1 once more we obtain an inequality such as (6). The preceding conclusion depends on the condition (B) and makes no use of the hypothesis (5); it clearly completes the proof of Theorem 6 .

\section{Consequences of (5) on the behavior of $\varphi_{m}\left(r e^{i \theta}\right)$ in $\beta<\left|\theta-\omega_{m}\right| \leqq \pi$.}

Lemma 11.1. Let the condition (5) be satisfied and let $s>1, \varepsilon>0$ and $\eta$ be given; it is assumed that $\eta$ is restricted by (8.1) and (8.3).

Then

$$
\frac{1}{2 \pi} \int_{\beta+2 \eta / 3}^{2 \pi-\beta-2 \eta / 3}\left|\Theta_{m}\left(r e^{i \theta}\right)\right| d \theta \leqq 32 s \varepsilon T\left(r_{m}\right) \quad\left(r \in I_{m}(s+1), m>m_{0}\right),
$$


and there are no more than

$$
q_{m}<\varepsilon T\left(r_{m}\right) \quad\left(m>m_{0}\right),
$$

zeros of $f(z)$ in the sector $\Delta_{m}$ (defined in (10.3)).

Proof: Lemma 7.2 is to be applied to $g_{m}(z)$ (defined by (8.6)). The parameter $\sigma$ of Lemma 7.2 will be chosen such that

$$
56 e^{(s+2) c} e^{\sigma(c-\mu)+(s+1) \mu}<\varepsilon \xi, \quad 2 \sigma>s+1 ;
$$

this is possible in view of (8.5) (a consequence of (5)).

Consider $g_{m}(z)$ in the sector

$$
\widetilde{\Delta}_{m}=\left\{z: r \in I_{m}(3 \sigma), \beta+\eta / 3 \leqq \theta \leqq 2 \pi-\beta-\eta / 3\right\},
$$

and let and let $\left\{\tilde{\eta}_{m}\right\}$ be chosen such that

$$
\tilde{\varphi}_{m}\left(r e^{i \theta}\right)=\varphi_{m}\left(r e^{i \theta}\right)-\tilde{\eta}_{m} T\left(r_{m}\right) \leqq 0 \quad\left(r e^{i \theta} \in \tilde{\Delta}_{m}, \tilde{\eta}_{m}>0, \tilde{\eta}_{m} \rightarrow 0\right) ;
$$

this is possible by $(2.12)$. [We remind the reader that (i) $\omega_{m}=0$; (ii) the parameter $m>m_{0}$ remains fixed throughout the following argument.]

Put

$$
\delta=\tilde{\eta}_{m} T\left(r_{m}\right), \quad B=3 T\left(r_{m}\right) .
$$

In order to justify our use of Lemma 7.2 we must verify that the conditions (7.9) and (7.10) hold. By (11.4)

$$
\varphi_{m}\left(r e^{i \theta}\right)=\log \left|g_{m}(z)\right| \leqq \tilde{\eta}_{m} T\left(r_{m}\right)=\delta \quad\left(z \in \tilde{\Delta}_{m}\right) .
$$

By (8.7) and (1.2)

$$
\begin{gathered}
m\left(r, 1 / g_{m}\right) \leqq\left\{2\left(r / r_{m}\right)^{\mu}+\eta_{m}\right\} T\left(r_{m}\right) \leqq\left(2 e^{(-\sigma+s+1) \mu}+\eta_{m}\right) T\left(r_{m}\right) \\
=e^{(-\sigma+s+1) \mu} T\left(r_{m}\right)\left(2+\eta_{m} e^{(\sigma-s-1) \mu}\right)<3 e^{(-\sigma+s+1) \mu} T\left(r_{m}\right) \\
\left(r_{m} e^{-\sigma-s-1} \leqq r \leqq r_{m} e^{-\sigma+s+1}, m>m_{0}\right),
\end{gathered}
$$

and hence our choice of $B$ in (11.5) leads to (7.10). By (7.12) we now find

$$
\begin{aligned}
-T\left(r_{m}\right)\left\{2 e^{\sigma c} \tilde{\eta}_{m}+6 e^{\sigma(c-\mu)+(s+1) \mu}\right\} \leqq \frac{1}{2 \pi} \int_{\beta+\eta / 3}^{2 \pi-\beta-\eta / 3} \log \left|g_{m}\left(r e^{i \theta}\right)\right| \cos (c(\theta-\pi)) d \theta \\
\left(r \in I_{m}(s+1)\right),
\end{aligned}
$$

and hence, by (11.3)

$$
\begin{aligned}
& -(\varepsilon \xi / 8) T\left(r_{m}\right) \leqq \frac{1}{2 \pi} \int_{\beta+\eta / 3}^{2 \pi-\beta-\eta / 3} \log \left|g_{m}\left(r e^{i \theta}\right)\right| \cos (c(\theta-\pi)) d \theta \\
& \left(r \in I_{m}(s+1), m>m_{0}\right) \text {. }
\end{aligned}
$$

In view of (11.6), in the above integrand,

$$
\log \left|g_{m}\right|-\delta \leqq 0,
$$


so that

$$
\begin{aligned}
& -(\varepsilon \xi / 8) T\left(r_{m}\right)-\delta / \pi c \leqq \frac{1}{2 \pi} \int_{\beta+2 \eta / 3}^{2 \pi-\beta-2 \eta / 3}\left\{\log \left|g_{m}\left(r e^{i \theta}\right)\right|-\delta\right\} \cos (c(\theta-\pi)) d \theta, \\
& -(\varepsilon \xi / 7) T\left(r_{m}\right) \leqq \frac{\xi}{2 \pi} \int_{\beta+2 \eta / 3}^{2 \pi-\beta-2 \eta / 3} \log \left|g_{m}\left(r e^{i \theta}\right)\right| d \theta \quad\left(r \in I_{m}(s+1), m>m_{0}\right) .
\end{aligned}
$$

To obtain (11.2) it is sufficient to combine (7.13), (11.3) and (11.7).

Assertion (1.18) of Lemma 1.1, applied to the polynomial $Q_{m}(z)$ of $(10.5)$, yields, in view of (11.2) and (11.7)

$$
-(\varepsilon / 7) T\left(r_{m}\right)-10 s \varepsilon T\left(r_{m}\right) \leqq \frac{1}{2 \pi} \int_{\beta+2 \eta / 3}^{2 \pi-\beta-2 \eta / 3} \Theta_{m}\left(r e^{i \theta}\right) d \theta \quad\left(r \in I_{m}(s+1), m>m_{0}\right),
$$

$$
\begin{aligned}
& \frac{1}{2 \pi} \int_{\beta+2 \eta / 3}^{2 \pi-\beta-2 \eta / 3}\left(\left\{-\Theta_{m}\left(r e^{i \theta}\right)\right\}^{+}+\Theta_{m}^{+}\left(r e^{i \theta}\right)\right) d \theta \\
& \leqq 11 s \varepsilon T\left(r_{m}\right)+\frac{1}{\pi} \int_{\beta+2 \eta / 3}^{2 \pi-\beta-2 \eta / 3} \Theta_{m}^{+}\left(r e^{i \theta}\right) d \theta .
\end{aligned}
$$

The definition (10.5), (11.4) and (1.18) show that the last integral in (11.8) cannot exceed

$$
\left(20 s \varepsilon+\tilde{\eta}_{m}\right) T\left(r_{m}\right) .
$$

Hence (11.8) implies (11.1) and Lemma 11.1 is proved. It may be noticed that the preceding proof only depends on the hypothesis (5) and that (10.4) and (10.6) (proved under different assumptions) have not been used.

12. Proof of Theorem 5. The assertions of Lemma 11.1 involve a fixed $\varepsilon>0$. A new diagonalization enables us to replace $\varepsilon$ by $\eta_{m}$ so that (11.1) and (11.2) take, respectively, the forms (10.6) and (10.4). Hence the proof of Theorem 5 follows from the reasoning at the end of Section 10.

13. Proof of the asymptotic symmetry. We apply Jensen's theorem three times: to $f(z)$ and to each of the two functions of the complex variable $\zeta$ :

$$
f\left(r_{m} e^{i\left(\omega_{m} \pm \beta\right)}(1+\zeta)\right) .
$$

We make certain simplifying assumptions which do not affect the result. We take

(i) $\omega_{m}=0$;

(ii) we assume that $f(z)$ has no poles in the sector defined by (9) and in its symmetrical;

(iii) $f\left(r_{m} e^{i \beta}\right) \neq 0, f\left(r_{m} e^{-i \beta}\right) \neq 0$.

The asymptotic formulae of Theorems 4 and 5 are invalid for certain values of $\theta$. We treat these values by an obvious use of Lemma III of [2]; the detailed reasoning will be omitted and simply indicated by the words "by the small arc lemma". 
To prove (11) we first deduce from (2), (3), (6) and the small arc lemma

$$
m(r, 1 / f) \leqq \delta T\left(r_{m}\right) \quad\left(r \in \Lambda(s), m>m_{0}(\delta)\right) ;
$$

the parameter $\delta>0$ may be chosen as small as we wish. Hence (by Jensen's formula)

$$
\liminf _{r \rightarrow \infty} \frac{N(r, 1 / f)}{T(r)} \geqq 1-\delta \quad(r \in \Lambda(s)) .
$$

Since $\delta>0$ is arbitrary

$$
N(r, 1 / f) \sim T(r) \quad(r \rightarrow \infty, r \in \Lambda(s))
$$

and (11) follows by the tauberian argument used in [A] to derive (17 [A]) from (16 [A]).

Put

$$
F(\zeta)=f\left(r_{m} e^{-i \beta}(1+\zeta)\right) \quad\left(\zeta=|\zeta| e^{i \varphi}\right)
$$

Let $n^{*}(x)$ be the number of zeros of $F(\zeta)$ in the disk $|\zeta|<x$ (by assumption $\left.n^{*}(0)=0\right)$. By Jensen's theorem

$$
\frac{1}{2 \pi} \int_{0}^{2 \pi} \log \left|F\left(t e^{i \varphi}\right)\right| d \varphi=\int_{0}^{t} \frac{n^{*}(x)}{x} d x+\log |F(0)|,
$$

and consequently

(13.3) $\frac{1}{2 \pi} \int_{0}^{2 \pi}\left\{\log \left|F\left(t e^{i \varphi}\right)\right|-\log \left|F\left(\sigma t e^{i \varphi}\right)\right|\right\} d \varphi \leqq n^{*}(t) \log (1 / \sigma) \quad(0<\sigma<1)$.

By assumption, the parameter $t$ is small enough to imply

$$
n^{*}(t) \leqq n^{-}\left(r_{m}(1+t)\right)-n^{-}\left(r_{m}(1-t)\right) .
$$

By (2), (3), (6) and the small arc lemma, we may given $\delta>0$, deduce

$$
\frac{1}{2 \pi} \int_{0}^{2 \pi} \log \left|F\left(t e^{i \varphi}\right)\right| d \varphi=\left(\mu T\left(r_{m}\right) / 2\right) \int_{0}^{\pi} \operatorname{Im}\left\{\left(1+t e^{i \varphi}\right)^{\mu}\right\} d \varphi+\eta\left(r_{m}, t\right) T\left(r_{m}\right),
$$

with the error term such that

$$
\left|\eta\left(r_{m}, t\right)\right| \leqq \delta \quad\left(m>m_{0}\right) .
$$

Hence, using (13.3) and (13.4), we find

$$
\begin{aligned}
& \frac{n^{-}\left(r_{m}(1+t)\right)-n^{-}\left(r_{m}(1-t)\right)}{T\left(r_{m}\right)} \geqq \frac{\mu}{2} \frac{1-\sigma}{\log (1 / \sigma)} \int_{0}^{\pi} \operatorname{Im}\left\{\frac{\left(1+t e^{i \varphi}\right)^{\mu}-\left(1+\sigma t e^{i \varphi}\right)^{\mu}}{1-\sigma}\right\} d \varphi \\
& -2 \delta / \log (1 / \sigma) \quad\left(m>m_{0}\right) \text {. }
\end{aligned}
$$

We let $m \rightarrow \infty$ with $\sigma$ and $\delta$ fixed. This yields a simple lower bound for

$$
\liminf _{m \rightarrow \infty} \frac{n^{-}\left(r_{m}(1+t)\right)-n^{-}\left(r_{m}(1-t)\right)}{T\left(r_{m}\right)}=B .
$$

We next let $\delta \rightarrow 0+$ and follow this by $\sigma \rightarrow 1-$. 
We thus find

$$
B \geqq(\mu / 2) \operatorname{Im}\left\{\int_{0}^{\pi} \mu t e^{i \varphi}\left(1+t e^{i \varphi}\right)^{\mu-1} d \varphi\right\}=(\mu / 2)\left\{(1+t)^{\mu}-(1-t)^{\mu}\right\} .
$$

Similarly, by symmetry,

$$
\liminf _{m \rightarrow \infty} \frac{n^{+}\left(r_{m}(1+t)\right)-n^{+}\left(r_{m}(1-t)\right)}{T\left(r_{m}\right)} \geqq(\mu / 2)\left\{(1+t)^{\mu}-(1-t)^{\mu}\right\} .
$$

If, in (13.5) or (13.6), it were impossible to replace liminf by lim, we would have

$$
\begin{gathered}
\limsup _{m \rightarrow \infty} \frac{\left\{n^{+}\left(r_{m}(1+t)\right)+n^{-}\left(r_{m}(1+t)\right)\right\}-\left\{n^{+}\left(r_{m}(1-t)\right)+n^{-}\left(r_{m}(1-t)\right)\right\}}{T\left(r_{m}\right)} \\
>\mu\left((1+t)^{\mu}-(1-t)^{\mu}\right) .
\end{gathered}
$$

In view of (4) and (7),

$$
n^{+}(u)+n^{-}(u)=n(u, 1 / f)-n\left(\xi r_{m}\right)+o\left(T\left(r_{m}\right)\right),
$$

uniformly as $m \rightarrow \infty$, and $u$ is confined to the intervals

$$
\xi r_{m}<u \leqq e^{s} r_{m} \quad(s>1) .
$$

Combining (11), (13.7) and (13.8) we obtain a contradiction which completes the proof of the asymptotic symmetry.

14. Proof of Theorem 7. Assume first that (5) is satisfied. Then (13.2) is valid and hence 0 is not a deficient value of $f(z)$. No other finite value may be deficient because we may treat $f(z)-\tau \quad(\tau=$ finite constant) exactly like $f(z)$. To justify this step we remark that if $f(z)$ satisfies the hypotheses ES so does $f(z)-\tau$. [It may clearly be necessary to modify the function $\eta(r)$ entering into the hypotheses ES.]

If (5) does not hold some $\tau \neq \infty$ may be deficient. Then, (3), (13) applied to $f(z)-\tau$, and the "small arc lemma" show that no finite $\tau^{\prime} \neq \tau$ may be deficient.

\section{References}

[1] EdreI, A., and W. H. J. Fuchs: The deficiencies of meromorphic functions of order less than one. - Duke Math. J. 27, 1960, 233-250.

[2] Edrei, A., and W. H. J. Fuchs: Bounds for the number of deficient values of certain classes of functions. - Proc. London Math. Soc., 3rd Ser., 13, 1962, 315-344.

[3] EdReI, A., and W. H. J. Fuchs: Asymptotic behavior of meromorphic functions with extremal spread I. - Ann. Acad. Sci. Fenn. Ser. A. I., 2, 1976, 67-111.

[4] Titchmarsh, E. C.: The theory of functions, 2nd ed. - Oxford, 1950.

Syracuse University

Department of Mathematics

Syracuse, New York 13210

USA
Cornell University

Department of Mathematics Ithaca, New York 14850 USA

Received 18 May 1976 